\title{
Economic and Feasibility Analysis for Stand-alone Solar Photovoltaic Generation System
}

\author{
Jeeng-Min Ling, Ping-Hsun Liu \\ Department of Electrical Engineering, Southern Taiwan University of Science and Technology, Tainan, Taiwan, China \\ Email: jmling@mail.stust.edu.tw, liushen@mail.njtc.edu.tw
}

Received April, 2013

\begin{abstract}
The paper presents a feasibility computing approach to solve the optimal planning problem applied to Stand-alone Photovoltaic (SPV) system by considering the reliability requirement and economical performance. Evaluation technique based on genetic algorithm to get global optimum capacity of solar array and battery in a SPV system is more efficiently. Explicit strategy selects proper values of systems' parameters improving local exploration and avoiding trapped in local optimum. Different requirements of system reliability are investigated to achieve the optimal planning of a SPV system. Sensitivity analysis of components' cost and load profiles are conducted to demonstrate the impacts of system uncertainty. The solar radiation and temperature data from the Central Weather Bureau of Taiwan at four different locations were used.
\end{abstract}

Keywords: Genetic Algorithms; Reliability; Optimization; Photovoltaic Systems

\section{Introduction}

Taiwan is located in the subtropical area and possesses excellent solar radiation for photovoltaic applications. Some new demonstration photovoltaic systems were installed on public buildings, such as the World Games 2009 Kaohsiung (1 MWp) and the National Museum of Taiwan History (195 KWp) etc. The government continues to promote the installation of solar photovoltaic systems through several projects, such as "Solar Top project", "Solar community Project" and "Solar Campus Project". It is believed that the installation capacity of solar photovoltaic systems will be boosted by the "Renewable Energy Development Bill” for future comercial applications [1].

SPV systems are becoming increasingly viable and cost-effective candidates for providing electricity to remote and offshore islands areas which operate at low capacity factors and where the gird extension is difficult and not economical. Issues concerning the security of supply and voltage rising in the micro-grid underline the need of storage system, like a stand-alone system, are becoming increasingly viable recently [2]. The planning of such an electrification unit requires an estimation of the capacities of photovoltaic (PV) module and battery (BTY) to satisfy a given load demand.

Some studies on sizing of the SPV system were studied [3-6]. The sizing method based on energy generation simulation for various numbers of PV and BTY capacity
(18 configurations) was presented using suitable models for the system devices [4]. The selection of the allocation of PV and BTY under corresponding reliability indices, the loss of load hours (LOLH) and the loss energy, should be considered the stochastic nature of both the radiation and the load demand. Based on the Borowy's and Salameh method [5], the system operation is simulated for various combination of PV and BTY sizes and the loss of power supply (LPSP) is calculated for each combination. For the desired LPSP, the PV versus BTY size are plotted to get the optimal solution, which minimizes the total system cost from the point on the sizing curve.

Several software tools are available for the design of stand-alone renewable energy systems [6-9]. The RET Screen International Clean Energy Decision Support Centre in Canada developed a decision making tool, RET Screen, to help planners to implement renewable energy and analyze the technical and financial viability of possible projects [7]. Hybrid 2 developed by the National Renewable Energy Laboratory in USA performs the detailed time series simulation of hybrid renewable systems [8]. The most popular simulation tool, Hybrid Optimization Model for Electric Renewable (HOMER) [9], uses hourly simulation to achieve optimal sizing of isolated renewable system. Most of the available software tools only identify and simulate a single design option; a range of possible design option is unavailable [6]. Furthermore, the impacts on the effects of non-linearity and op- 
timization in system model, and the variations of the significant design variables is needed to investigate the usefulness of these simulation and optimization tools applied to specific applications.

The paper proposes a procedure based on genetic algorithms (GA) to get global optimum capacity of PV array and battery in a SPV system under compromise between the reliability and total installed cost. Compared with the conventional Lagrangian relaxation optimization, GA approach finds the global optimum more efficiently. The sensitivity analysis for the component cost and load profiles were also discussed to show the impacts of the optimal results of planning. The optimal sizing of a SPV system incorporating solar resources uncertainty is also considered in the aspects of long-term planning. The real solar radiation/temperature data from four weather stations have been tested to simulate the practicability of planning results for a SPV system.

\section{The Optimal Design Method}

In the design and planning of stand-alone renewable energy systems, the optimal sizing is an important and challenging task as the coordination among renewable energy resources, generators, storage capacity and it's complicated load.

\subsection{System Modeling}

A SPV system consists of solar array, battery bank, control and power converting components. The PV-array convert's sun light into DC electricity. PV array is made up of several interconnected PV modules. The batteries store the electrical energy for use when needed. The block diagram of the proposed system is shown in Figure 1.

\subsection{The Reliability Analysis of a SPV Generation}

To access the available solar generation of a PV system in candidate region is one of the most important parameters before installation. Because of the intermittent solar radiation characteristics, which highly influence the resulting energy production, reliability analysis has been

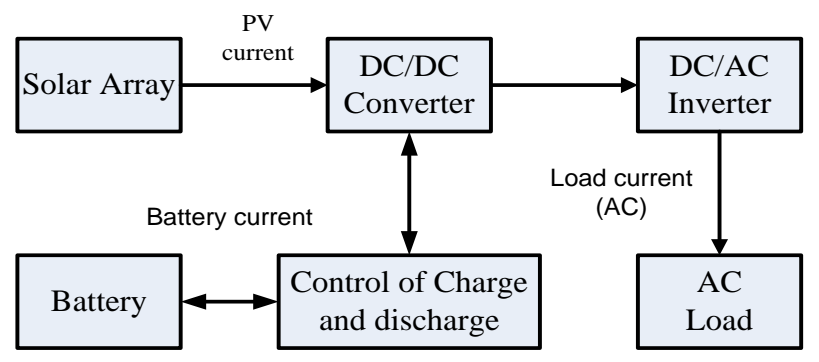

Figure 1. Block diagram of the SPV system. considered as an important step in any planning and design process. In the paper, the reliability index evaluated is the total loss of load hours (LOLH) which is the summation of loss of load expectation events expressed in hours over a specified time (usually one year). At these time, a SPV system is unable to meet the load requirements due to lack of power at an instant.

LOLH is a feasible measure to system performance for assumed or known load distribution. Zero LOLH means that the load will always be satisfied. Larger LOLH implies the customer will be suffered from a higher probability of losing power. It is a popular demand side index in the system planning. LOLH can be defined by the following equation:

$$
\begin{aligned}
& L O L H=\sum_{i=1}^{n} \sum_{j=1}^{24} f(i, j) \\
& f(i, j)=\left\{\begin{array}{cc}
0 & \text { if }(S(i, j)-L(i, j)) \geq S_{\min } \\
1-\frac{S(i, j)-S_{\min }}{L(i, j)} & \text { if } S(i, j)>S_{\min } \text { a } n S(i, j-1)>S_{\text {min }} \\
1 & \text { if }(S(i, j)-L(i, j))<S_{\min } \text { o } \quad \mathcal{S}(i, j-1)<S_{\min }
\end{array}\right.
\end{aligned}
$$

where hour,

$S(i, j)$ : the capacity state of BTY in the $i$ th day- $j$ th $L(i, j)$ : the consumed load in the $i$ th day- $j$ th hour, $f(i, j)$ : system shortage in the ith day- jth hours,

$S_{\min }$ : Minimum battery discharge capacity.

The amount of solar radiation determines the current output of a PV generation. After considering load profile, the output power of a PV generation can be conducted to evaluate the charge/discharge current $I_{b}$ of BTY. Two main directions of $I_{b}$ lead to different operation modes of SPV: positive $I_{b}$ is the mode of $\mathrm{PV}$ generation greater than load consumption, while negative $I_{b}$ induced by the shortage of a SPV generation. At this mode, the state of charge and minimum battery discharge capacity of BTY should be integrated to calculate the LOLH. The Summary of a LOLH table over a specified time (one year) associated with different combinations of PV/BTY capacity allocations, i.e. specific reliability constrain, can be constructed to form the constrained optimization.

\subsection{The Constrained Optimization Formulation}

The optimal size problem of a SPV system belongs to a constrained optimization. The optimum achieves at the best compromise between system power reliability and cost. The objective function of the proposed system can be expressed as the installed cost:

$$
C=C_{w} \times P V+C_{b} \times B T Y+C_{i}
$$

where

$C$ : the total cost for installed a SPV system,

$C_{i}$ : the initial cost for system installation, 
$P V, B T Y$ : the capacity of solar array and battery,

$C_{W}, C_{b}$ : the unit cost of PV $\left(\$ / \mathrm{W}_{\mathrm{p}}\right)$ and BTY $(\$ / \mathrm{Wh})$.

Constraint function has been produced for eight values of LOLH, 0, 10, 20, 50, 100, 150, 200 and 400 hours, in terms of the given load consumption. Different requirement of systems' reliability can be evaluated by selecting the suitable simulation range of PV and BTY capacity. It is significant for a SPV planner to get options under different system shortages.

\subsection{Optimization Technique using GA}

Genetic algorithm (GA) is an population based search and optimization technique. It has been developed to imitate the process of natural evolutionary of genetics $[10,11]$. GA takes selection, crossover and mutation to imi- tate evolution processes. The selection evaluation is to determine the chosen chromo some, each chromosome consists of two genes. Specific values of LOLH with allocation of PV and BTY in a LOLH table pass the selection evaluation via the fitness cost function. If the evaluation of qualified chromosome has a lowest total cost of a SPV system than the cost obtained at the previous iterations, the size of PV/BTY allocation was considered to be the optimal solution for the constrained optimization in this iteration. The optimal solution will be replaced by better solution, if any, produced in next GA generations [12]. The optimal solution will then be sub- ject to the process of crossover and mutation, it produces the next generation have been reached. The iteration will continue when convergence criterion satify.

The flowchart of the optimization process is illustrated in Figure 2. If any of the initial population chromosomes violates the system constraint, it is replaced by a new chromosome. The PV array current output is calculated according to the PV system model by using the specifications of the PV module, ambient temperatures and solar radiation conditions. The battery capacity is permitted to discharge up to a limit defined by the maximum depth of discharge, which is specified by the system designer at the beginning of the optimal capacity process.

The GA was implemented by Matlab ${ }^{\circledR}$ and employed the operators of roulette-wheel selection, single-point crossover, single-bit mutation, and elite replacement. The following parameters are used in the GA simulation,

- The population size: 200

- Generation: 50

- Mutation rate: 0.01

\section{Analysis of Reliability Simulation}

The optimal size of a SPV system at four selected sites of weather station in Taiwan were investigated and compared. The combination of different PV/BTY capacity has 3200 various states to evaluate each degree of LOLH per year. Using the real meteorological data of year 2008 at Tainan weather station, the possible combination of PV/BTY size associated with different LOLH values can be depicted by the three dimensional (3D) curve shown in the Figure 3(a). Eight specified values of LOLH (0, 10, 20, 50, 100, 150, 200 and 400 hours) are selected and depicted by eight curves in the two dimensional (2D) distribution with different colors in Figure 3(b).

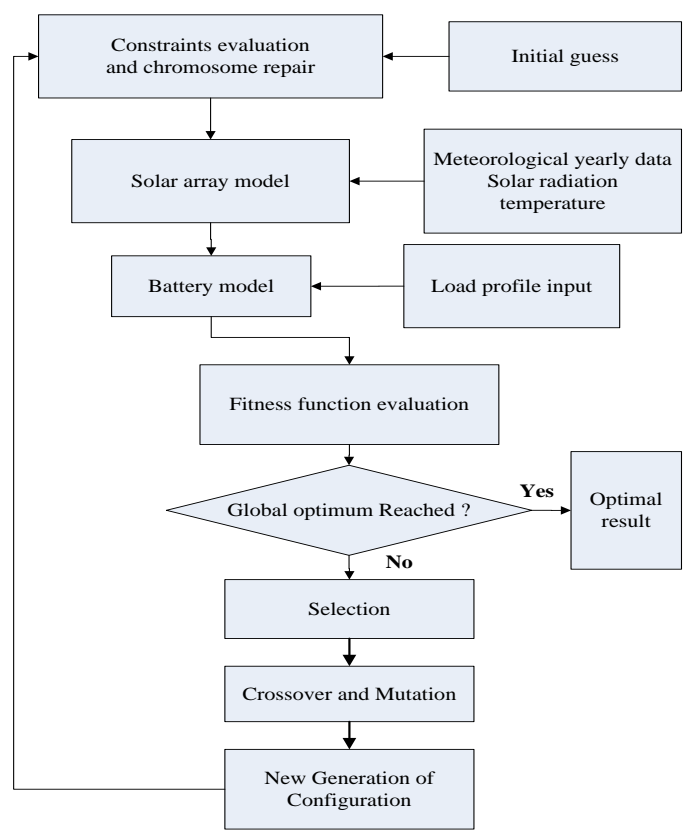

Figure 2. Flowchart of the optimal sizing model using GA.

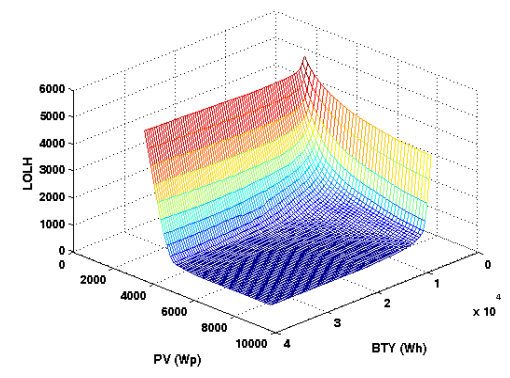

(a) 3D distribution

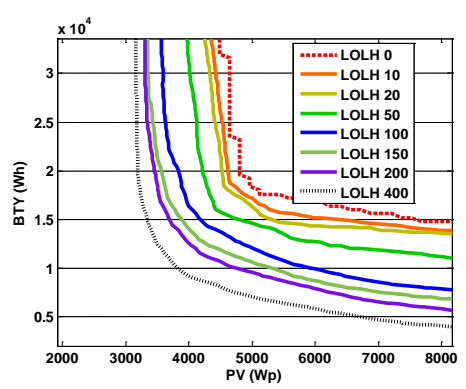

(b) $2 \mathrm{D}$ distribution

Figure 3. Reliability curves for different combinations of PV/BTY capacity with different LOLH values at Tainan. 
Each 2D curve indicates the trend of PV/BTY size changing with a constant system shortage. The different combinations of PV/BTY capacity which meet the same reliability degree of power supply can be expressed by plotting the 2D trade-off curve. In these 2D trade-off curves, the upper most curve belongs to LOLH $=0$, while the lowest curve occurs when the system shortage hour is 400 hours.

The optimal size of a SPV system can be affected the location because of different solar radiation. In order to clarity the influence of location, the meteorological data from four different weather stations in the Central Weather Bureau of Taiwan were simulated. Figure 4 shows these results.

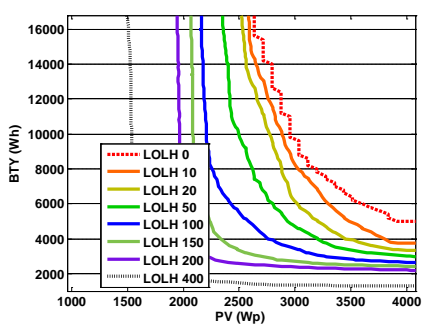

(a) Chiayi

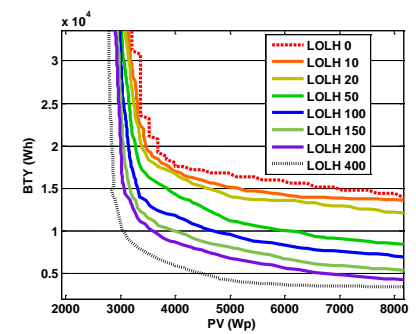

(b) Tainan

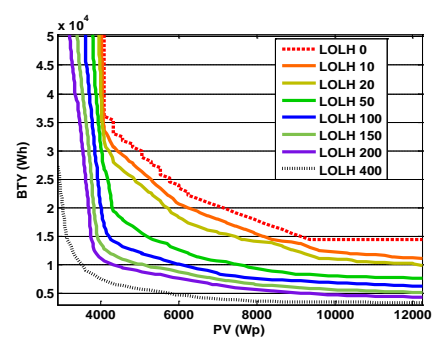

(d) Dongjitao

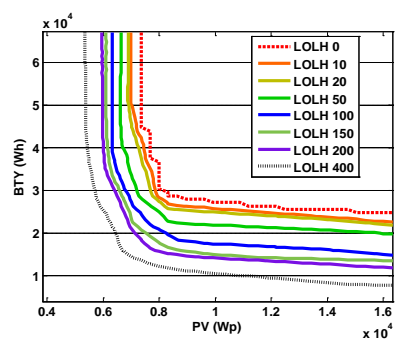

(f) Anpu

Figure 4. PV/BTY curves for different LOLH degree at four locations for Actual load.
Using the eight LOLH curves shown in Figure 4, the influence of LOLH on the planning of PV/BTY capacity of a SPV system can be identified. Considerable installed PV and BTY capacity reductions occur as LOLH varies from 0 to 400 hour. The Anpu site which has the poorest solar radiation among these four tested sites causes the installed capacity of PV/ BTY to be very large. On the other side, the sites at Chiayi and Tainan characterize a much richer solar radiation result implying to a smaller installed capacity.

The LOLH curve shown in Figure 4 can be roughly divided into two blocks. In the left/vertical block, increasing of smaller PV installed capacity lead to a remarkable BTY capacity reduction, especially at the site of Anpu. In the right/horizontal block, the BTY capacity decreases gradually with larger increasing of PV installed capacity. The optimum occurs at the turning point of a LOLH curve, i.e. the overlapping part of these two blocks.

Analysis of the relationship of PV/BTY capacity in terms of LOLH can determine the optimal capacity allocation status. Due to the unit cost of a PV component is much larger than that of BTY, the total installation cost of PV significantly dominates the final optimal cost. A system with a large PV size and small BTY size can be prone to quite fast charging/discharging of the batteries. For this case, the reliability of system would be largely dependent on the solar radiation alone. Such design exposes the system to instantaneous variations in the solar radiation. Even though the LOLH can be reduced by providing a larger PV size, the degree of LOLH may be quite high depending upon the radiation characteristics. On the other side, a system with a small PV size and large BTY size will result in slower charging/discharging rates in the batteries. Most of the converted energy will be stored when the instantaneous generation is in excess of the load. This LOLH in the design may be large due to a small solar array size.

\section{Analysis of Optimal Sizing Simulation}

\subsection{Influence of Different Load Profile and Reliability Requirement}

The proposed optimal algorithm was implemented by Matlab. The real solar radiation/temperature data from the central weather Bureau of Taiwan on the year 2003 to 2009 have been simulated. The influences of four different load profiles, constant, peak, sinusoidal and actual load, are investigated. It is noted that the actual load is the power profile of a laboratory located at the A building of Southern Taiwan University of Science and Technology shown in Figure 5. Daily average of the actual load for testing is $10.68 \mathrm{~kW}$. Using this value as the benchmark, three other load profiles, constant, peak and 
sinusoidal, can be evaluated to investigate the impacts result from different load profiles during SPV system planning. This optimal result of PV/BTY corresponds to the following hours of system shortage, e.g., 0, 10, 20, 50, 150, 200 and 400 as shown in Table 1.

It can be found that the optimal cost for installation is sensitive to the desired system reliability. The installed cost of a SPV system will increase to meet the desired higher system reliability requirement, i.e., LOLH is inversely proportional to system cost. As for the influence of load profile, we can verify the phenomenon that the installation cost of a SPV implemented in actual load is lower than other three load types at four testing sizes because of the diversity of load profile. The highest cost often occurs in the sinusoidal load, it concentrates the load consumption in one half of period time.

Influence of locations to the optimal cost can be con- cluded by the characteristics of solar radiation. Chiayi region has the lowest total installation cost with its good solar radiation and ambient temperature average.

Conversely, Anpu has the highest installed cost compared with other regions for the same reason.

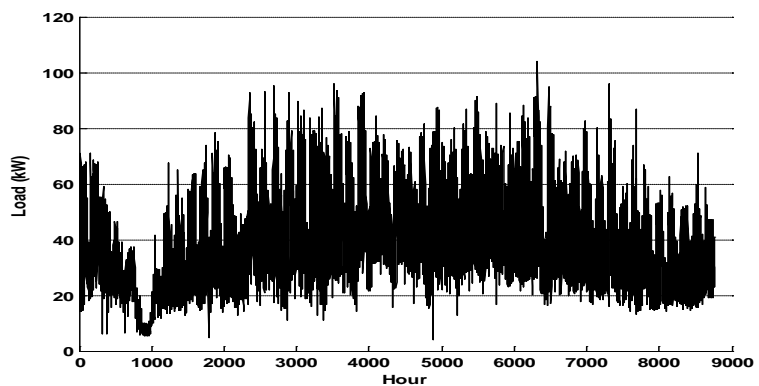

Figure 5. Hourly profile of testing load.

Table 1. The optimal size of a spv at 4 sites under four different load patterns.

(a) Chiayi

\begin{tabular}{ccccccccccccc}
\hline Chiayi & \multicolumn{3}{c}{ Fixed load } & \multicolumn{3}{c}{ Peak load } & \multicolumn{3}{c}{ Sinusoidal load } \\
\hline $\begin{array}{c}\text { LOLH } \\
\text { (hours) }\end{array}$ & PV (Wp) & BTY (Wh) & Cost (USD) & PV (Wp) & BTY (Wh) & Cost (USD) & PV (Wp) & BTY (Wh) & Cost (USD) & PV (Wp) & BTY (Wh) & Cost (USD) \\
\hline 0 & 3913.7 & 12612.4 & 21505.2 & 3909.5 & 15114.2 & 21971.9 & 3910.0 & 13778.2 & 21714.1 & 2871.5 & 11397.0 & 16195.7 \\
10 & 3896.4 & 11702.7 & 21243.6 & 3893.0 & 14864.1 & 21842.6 & 3884.5 & 13380.2 & 21512.5 & 2824.1 & 10788.8 & 15846.7 \\
20 & 3945.1 & 9107.1 & 20975.3 & 3865.5 & 14934.8 & 21722.6 & 3875.9 & 12791.9 & 21356.1 & 2748.2 & 10320.1 & 15386.0 \\
50 & 3783.6 & 8767.5 & 20123.2 & 3735.6 & 14996.6 & 21102.6 & 3782.1 & 9367.0 & 20232.5 & 2495.4 & 9982.8 & 14089.6 \\
100 & 3462.9 & 10170.2 & 18835.1 & 3753.0 & 8606.3 & 19942.7 & 3243.2 & 11375.0 & 18000.6 & 2240.5 & 8413.8 & 12543.7 \\
150 & 3145.3 & 10998.3 & 17450.6 & 3442.0 & 9359.4 & 18575.7 & 2916.8 & 8865.8 & 15923.1 & 2145.9 & 6488.5 & 11708.3 \\
200 & 2900.0 & 10225.6 & 16106.3 & 3369.9 & 7420.5 & 17847.3 & 2647.5 & 8786.2 & 14597.1 & 2065.2 & 3364.7 & 10707.0 \\
400 & 2427.1 & 4377.9 & 12665.7 & 2580.6 & 2625.2 & 13072.0 & 2016.1 & 3423.9 & 10479.9 & 1602.7 & 2501.0 & 8287.8 \\
\hline
\end{tabular}

(b) Tainan

\begin{tabular}{|c|c|c|c|c|c|c|c|c|c|c|c|c|}
\hline \multirow{2}{*}{$\begin{array}{l}\text { Tainan } \\
\begin{array}{l}\text { LOLH } \\
\text { (hours) }\end{array}\end{array}$} & \multicolumn{3}{|c|}{ Fixed load } & \multicolumn{3}{|c|}{ Peak load } & \multicolumn{3}{|c|}{ Sinusoidal load } & \multicolumn{3}{|c|}{ Actual load } \\
\hline & PV (Wp) & BTY (Wh) & Cost (USD) & PV (Wp) & BTY (Wh) & Cost (USD) & PV (Wp) & BTY (Wh) & Cost (USD) & PV (Wp) & BTY (Wh) & Cost (USD) \\
\hline 0 & 3558.5 & 18036.9 & 20832.3 & 3655.0 & 21556.7 & 21987.3 & 3606.2 & 18716.9 & 21196.9 & 3515.0 & 21456.0 & 21286.1 \\
\hline 10 & 3587.0 & 16118.5 & 20597.4 & 3715.6 & 16879.3 & 21371.3 & 3600.4 & 18103.3 & 21049.2 & 3409.3 & 20956.6 & 20674.3 \\
\hline 20 & 3618.5 & 15421.0 & 20615.2 & 3622.7 & 15629.5 & 20676.1 & 3657.9 & 14596.0 & 20646.4 & 3298.9 & 20789.3 & 20104.6 \\
\hline 50 & 3528.6 & 13967.3 & 19894.3 & 3663.4 & 15284.7 & 20806.8 & 3568.9 & 14185.0 & 20133.0 & 3247.2 & 19599.6 & 19621.3 \\
\hline 100 & 3454.4 & 13122.1 & 19368.5 & 3620.8 & 16108.7 & 20760.2 & 3481.0 & 13112.4 & 19496.1 & 3290.9 & 15090.1 & 18955.8 \\
\hline 150 & 3375.4 & 12404.8 & 18844.5 & 3612.0 & 14715.7 & 20446.0 & 3386.1 & 12291.3 & 18874.3 & 3165.1 & 15155.8 & 18356.3 \\
\hline 200 & 3281.8 & 12314.1 & 18371.3 & 3455.8 & 17170.2 & 20163.7 & 3280.0 & 11758.9 & 18254.6 & 3027.5 & 15792.7 & 17810.7 \\
\hline 400 & 3060.4 & 10631.6 & 16966.2 & 3262.9 & 14874.9 & 18777.7 & 3014.3 & 10708.7 & 16756.8 & 2946.3 & 10736.0 & 16431.2 \\
\hline \multicolumn{13}{|c|}{ (c) Anpu } \\
\hline Anpu & \multicolumn{3}{|c|}{ Fixed load } & \multicolumn{3}{|c|}{ Peak load } & \multicolumn{3}{|c|}{ Sinusoidal load } & \multicolumn{3}{|c|}{ Actual load } \\
\hline $\begin{array}{l}\text { LOLH } \\
\text { (hours) }\end{array}$ & PV (Wp) & BTY (Wh) & Cost (USD) & PV (Wp) & BTY (Wh) & Cost (USD) & PV (Wp) & BTY (Wh) & Cost (USD) & PV (Wp) & BTY (Wh) & Cost (USD) \\
\hline 0 & 15589.1 & 71962.0 & 89888.9 & 15589.1 & 71962.0 & 89888.9 & 15602.5 & 74994.8 & 90544.3 & 7777.5 & 31230.7 & 43936.6 \\
\hline 10 & 15416.4 & 70954.2 & 88851.8 & 15416.4 & 70954.2 & 88851.8 & 14921.4 & 70978.2 & 86447.4 & 7473.5 & 29880.6 & 42194.1 \\
\hline 20 & 15164.4 & 71332.6 & 87699.1 & 15164.4 & 71332.6 & 87699.1 & 14806.9 & 70079.7 & 85714.7 & 7210.6 & 29149.6 & 40771.9 \\
\hline 50 & 13538.2 & 63854.2 & 78327.6 & 13538.2 & 63854.2 & 78327.6 & 13600.8 & 64959.3 & 78847.5 & 6697.7 & 38271.3 & 40051.4 \\
\hline 100 & 12301.0 & 56919.5 & 70955.4 & 12301.0 & 56919.5 & 70955.4 & 12290.6 & 56019.9 & 70730.0 & 6395.1 & 35393.9 & 38018.4 \\
\hline 150 & 11729.7 & 42473.4 & 65362.0 & 11729.7 & 42473.4 & 65362.0 & 11284.0 & 46565.0 & 63989.4 & 5923.7 & 40131.1 & 36646.0 \\
\hline 200 & 11053.5 & 35342.3 & 60682.3 & 11053.5 & 35342.3 & 60682.3 & 10485.3 & 35775.6 & 58001.1 & 6152.1 & 33338.1 & 36435.4 \\
\hline 400 & 7552.1 & 43976.5 & 45321.1 & 7552.1 & 43976.5 & 45321.1 & 6849.1 & 40783.1 & 41277.6 & 5594.9 & 31115.5 & 33290.4 \\
\hline
\end{tabular}




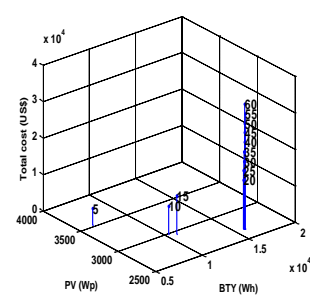

(a) Chiayi

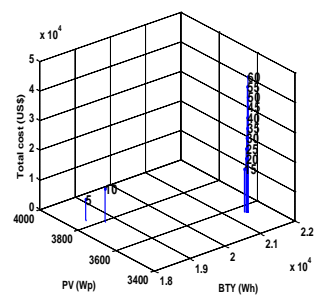

(b) Tainan

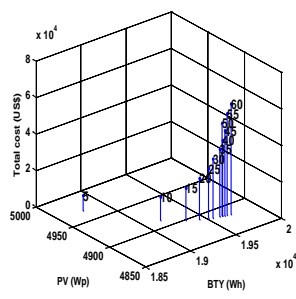

(c) Tawu

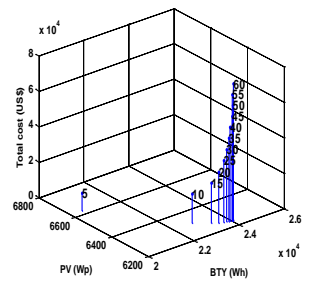

(d) Lanyu

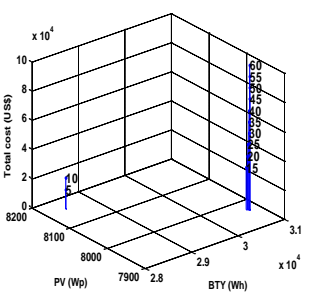

(e) Anpu

Figure 6. The optimal sizing curve with different ratio of to PV/BTY cost ( LOLH=0, the actual load).

Table 2. The optimal sizing results with different ratio of pv/bty cost under actual load.

\begin{tabular}{|c|c|c|c|c|c|c|c|c|c|c|c|c|c|c|c|}
\hline \multirow{2}{*}{$\begin{array}{l}\text { Cost } \\
\text { ratio }\end{array}$} & \multicolumn{3}{|c|}{ Chiayi } & \multicolumn{3}{|c|}{ Tainan } & \multicolumn{3}{|c|}{ Tawu } & \multicolumn{3}{|c|}{ Lanyu } & \multicolumn{3}{|c|}{ Anpu } \\
\hline & $\begin{array}{c}\text { PV } \\
\text { (Wp) }\end{array}$ & $\begin{array}{l}\text { BTY } \\
\text { (Wh) }\end{array}$ & $\begin{array}{c}\text { Cost } \\
\text { (US\$) }\end{array}$ & $\begin{array}{c}\text { PV } \\
\text { (Wp) }\end{array}$ & $\begin{array}{l}\text { BTY } \\
\text { (Wh) }\end{array}$ & $\begin{array}{l}\text { Cost } \\
\text { (US\$) }\end{array}$ & $\begin{array}{c}\text { PV } \\
\text { (Wp) }\end{array}$ & $\begin{array}{l}\text { BTY } \\
\text { (Wh) }\end{array}$ & $\begin{array}{l}\text { Cost } \\
\text { (US\$) }\end{array}$ & $\begin{array}{c}\text { PV } \\
\text { (Wp) }\end{array}$ & $\begin{array}{l}\text { BTY } \\
\text { (Wh) }\end{array}$ & $\begin{array}{l}\text { Cost } \\
\text { (US\$) }\end{array}$ & $\begin{array}{c}\text { PV } \\
\text { (Wp) }\end{array}$ & $\begin{array}{l}\text { BTY } \\
\text { (Wh) }\end{array}$ & $\begin{array}{c}\text { Cost } \\
\text { (US\$) }\end{array}$ \\
\hline 10 & 2984.2 & 10286.6 & 7812.9 & 3793.91 & 18724.6 & 11032.2 & 4911.9 & 19164.4 & 13294.5 & 6309.2 & 22826.6 & 16727.9 & 8161.1 & 28403.1 & 21419.2 \\
\hline 15 & 2940.5 & 10811.2 & 10692.3 & 3558.22 & 21411.6 & 14560.3 & 4897.9 & 19337.0 & 18068.8 & 6266.4 & 23335.2 & 22843.8 & 7968.7 & 30737.6 & 29256.4 \\
\hline 25 & 2667.9 & 15850.9 & 16071.9 & 3556.02 & 21453.5 & 21485.2 & 4888.8 & 19510.5 & 27594.3 & 6246.3 & 23714.7 & 35020.2 & 7967.3 & 30764.4 & 44769.4 \\
\hline 30 & 2666.6 & 15884.1 & 18668.1 & 3555.52 & 21465.9 & 24946.5 & 4887.4 & 19548.5 & 32352.6 & 6243.2 & 23800.8 & 41099.3 & 7966.8 & 30777.5 & 52524.9 \\
\hline 35 & 2666.7 & 15881.7 & 21264.1 & 3555.32 & 21473.4 & 28407.5 & 4885.6 & 19606.5 & 37109.4 & 6240.8 & 23878.9 & 47175.7 & 7966.6 & 30784.3 & 60280.3 \\
\hline 40 & 2665.8 & 15911.3 & 23858.7 & 3555.22 & 21475.3 & 31868.4 & 4884.9 & 19635.0 & 41865.2 & 6239.2 & 23938.3 & 53250.3 & 7966.5 & 30786.8 & 68035.5 \\
\hline 45 & 2665.3 & 15934.8 & 26453.5 & 3554.92 & 21488.1 & 35329.0 & 4884.4 & 19656.2 & 46620.3 & 6237.6 & 24008.3 & 59324.0 & 7966.3 & 30792.8 & 75790.6 \\
\hline 50 & 2665.1 & 15945.1 & 29048.2 & 3554.82 & 21493.7 & 38789.6 & 4885.1 & 19627.2 & 51376.1 & 6237.2 & 24030.6 & 65396.4 & 7966.3 & 30795.5 & 83545.6 \\
\hline 55 & 2664.6 & 15981.7 & 31645.0 & 3555.02 & 21484.2 & 42250.3 & 4884.0 & 19675.0 & 56129.4 & 6237.6 & 24010.2 & 71468.4 & 7966.3 & 30797.7 & 91300.6 \\
\hline 60 & 2665.1 & 15944.2 & 34237.0 & 3554.72 & 21497.3 & 45710.7 & 4883.3 & 19713.2 & 60883.2 & 6236.6 & 24065.2 & 77539.5 & 7966.3 & 30797.5 & 99055.5 \\
\hline
\end{tabular}

\subsection{Influence of Component Cost Variations}

The variations of component cost for a PV system is uncertain. In this study, the unit cost of a PV is set to be the range of $4.67 \sim 5.61$ (USD/Wp), and a BTY is 0.093 0.280 (USD/Wh). The base cost of BTY capacity is set to 0.1947 (USD/Wh) for demonstration. A feasible range of cost ratio will be tested by $5 \sim 60$, it can be represented as the ratio of $C_{w}$ to $C_{b}$ shown in equation 2 .

12 discrete values of cost ratio changing from 5 to 60 with increment 5 were used to show its effects. The following simulation is derived from LOLH equals to 0 . The optimal size in terms of different component cost ratios are depicted in Figure 6 and Table 2. As shown in Figure 6, the optimal size of PV and BTY is insensitive to the changing of cost ratio when its value greater than 10 to 15 . It is believed that the value of cost ratio to be smaller than 20 is unreasonable. In some senses, it means explicitly the robust of the optimal results regardless of price fluctuation. On the other side, a installed cost increase proportionally when the cost ratio grows.

Results show the optimal result regional dependence. Different pattern of optimal size appears in different region. The highly regional feature for the planning of a renewable system should be identified. Challenge from volatility and spatial diversity of solar resource is another issue. The optimal size of a SPV system is obviously reduced when the quality of the solar resources increasing.

\section{Conclusions}

At different regions with various meteorological conditions and solar energy reserves, the electric power production from renewable energy is highly unreliable and unpredictable. Well-designed system is a basic requirement for any system planner. In the paper, Different requirements of system reliability are conducted statisticcally to achieve the optimal capacity allocation for a SPV system. Variations resulted from the cost of SPV component and load amount are investigated to satisfy the specific reliability requirement to demonstrate the impacts of system uncertainty in the long-term planning.

The optimal size of a SPV system is found efficiently by a GA optimization technique. Global optimum with relative computation simplicity has been attained. The simulation results of this paper is believed to be a worthy reference for decision-making can be considered as im- 
portant references of the photovoltaic generation installation.

\section{Acknowledgements}

The authors would like to thank the financial support of National Science Council under grant number NSC101-2221-E-218-043.

\section{REFERENCES}

[1] J. J. Hwang, "Promotional Policy for Renewable Energy Development in Taiwan," Renewable and Sustainable Energy Reviews, Vol. 14, No. 3, 2010, pp. 1079-1087. doi:10.1016/j.rser.2009.10.029

[2] R. Hara, et al., "Testing the Technologies: Demonstration Grid-connected Photovoltaic Projects in Japan,” IEEE Power and Energy Magazine, Vol. 7, No. 3, May/June 2009, pp. 77-85.

[3] W. X. Shen, "Optimally Sizing of Solar Array and Battery in Standalone Photovoltaic System in Malaysia,” Renewable Energy, Vol. 34, No.1, 2009, pp. 348-352. doi:10.1016/j.renene.2008.03.015

[4] G. B. Shrestha and L. Goel, "A Study on Optimal Sizing of Stand-alone Photovoltaic Stations,” IEEE Transactions on Energy Conversion, Vol.13, No. 4, 1998, pp.373-378. doi:10.1109/60.736323

[5] S. B. Borowy and Z. M. Salameh, "Methodology for Op- timal Sizing of the Combination of a Battery Bank and PV Array in a Wind/PV Hybrid System,” IEEE Transactions on Energy Conversion, Vol. 11, No. 2, 1996, pp.367-375.

[6] Jose L. Bernal-Agustin and R. Dufo-Lopez, "Simulation and Optimization of Stand-alone Hybrid Renewable Energy Systems," Renewable and Sustainable Energy Reviews, Vol. 13, No. 8, 2009, pp. 2111-2118.

[7] RETS, 2004. Available: http://www.retscreen.net/ang/t_software.php

[8] RERL, 2007. Available: http://www.ceere.org/rerl/projects/software/hybrid2/Hy2_ users_manual.pdf.

[9] Homer, The Hybrid Optimization Model for Electric Renewable, Available: http://www.nrel.gov/homer/includes/downloads/HOMER Brochure_English.pdf.

[10] J. Holland, Adaptation in Natural and Artificial Systems: The University of Michigan. 1975

[11] P. Arun, R. Banerjee and S. Bandyopadhyay, "Optimal sizing of Photovoltaic Battery Systems Incorporating Uncertainty through Design Space Approach," Solar Energy, Vol. 83, No.7, 2009, pp.1013-1025. doi:10.1016/j.solener.2009.01.003

[12] Y. X. Hong, Z. Wei, L. Lin and F. H. Zhao, "Opimal Sizing Method for Stand-alone Hybrid Solar-wind System with LPSP Technology by using Genetic Algorithm," Solar Energy, 2008, pp. 354-367. 